\title{
Editorial
}

\section{Advanced Topics in Dynamics of Complex Systems}

\author{
Dumitru Baleanu, ${ }^{1}$ Bashir Ahmad, ${ }^{2}$ Juan J. Nieto, ${ }^{3}$ and J. A. Tenreiro Machado ${ }^{4}$ \\ ${ }^{1}$ Department of Mathematics and Computer Sciences, Cankaya University, Balgat, 06530 Ankara, Turkey \\ ${ }^{2}$ Department of Mathematics, Faculty of Science, King Abdulaziz University, P.O. Box 80203, Jeddah 21589, Saudi Arabia \\ ${ }^{3}$ Institute of Mathematics, University of Santiago de Compostela, 15782 Santiago de Compostela, Spain \\ ${ }^{4}$ Department of Electrical Engineering, Institute of Engineering, Polytechnic of Porto (ISEP), Rua Dr. Antonio Bernardino de Almeida, \\ 431, 4200-072 Porto, Portugal \\ Correspondence should be addressed to Dumitru Baleanu; dumitru.baleanu@gmail.com
}

Received 23 February 2014; Accepted 23 February 2014; Published 23 March 2014

Copyright (C) 2014 Dumitru Baleanu et al. This is an open access article distributed under the Creative Commons Attribution License, which permits unrestricted use, distribution, and reproduction in any medium, provided the original work is properly cited.

The investigation of complex systems in a unified manner is recognized during the last few years as a new and very promising scientific discipline. Therefore, a wide range of interdisciplinary methods and techniques should be applied and, at the same time, new tools should be invented to extract hidden and valuable information from the dynamics of complex systems.

The main aim of this special issue is to present algorithms and modelling techniques for analysing complex phenomena and dynamical systems with nonlinear and memory effects.

Fractional calculus, in view of its practical applications in several disciplines such as physics and engineering problems, is found to be capable of describing phenomena possessing long range memory effects that are difficult to handle with classical integer-order calculus. There has been a growing interest in the adaptation of fractional calculus as an effective modeling tool for complex systems, giving rise to new perspectives in their dynamical analysis and control. This development in the scientific knowledge is demonstrated by a large number of books and events emerging on the topic during the past years. However, many phenomena still pose considerable challenges to the concerned community and fractional calculus seems to be the probable candidate to encompass broader paradigms by shedding light into the description of complexity.

This special issue contains the papers addressing the recent theoretical advances and experimental results on the topics such as chaos, fractals, fractional dynamics, impulsive and neural systems, information and computation theory, signal processing (filtering, restoration, reconstruction, and analysis of fractal noise), image encryption and hiding, mechanics, Navier-Stokes equations on Cantor sets, mechatronics (viscoelasticity, vibration insulation, and control), biology (orthodontic root resorption, classifications of some structural domains of proteins, and genetic algorithms), biophysics (signal and models of biological systems, control of language of competitive models, tourism systems, and viscoelasticity in biology), bioengineering, nanotechnology, anomalous diffusion, and complex adaptive systems.

The Guest Editors believe that the set of selected advanced studies offer readers a timely update of important research topics and may serve as a platform for motivating further contributions in this fast evolving arena.

\section{Acknowledgments}

The Guest Editors of this special issue would like to express their gratitude to the authors who have submitted papers for consideration. Thanks also to the many individuals who served as referees of the submitted papers. All the participants have made it possible to have a very stimulating interchange of ideas. We hope that these topics will stimulate future research. We would also like to thank the editorial board members of this journal for their support and help throughout the preparation of this special issue.

Dumitru Baleanu Bashir Ahmad Juan J. Nieto 


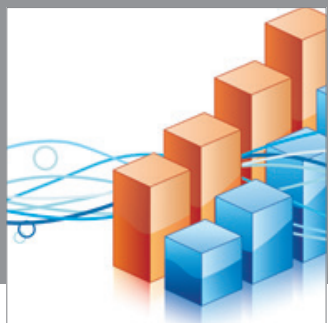

Advances in

Operations Research

mansans

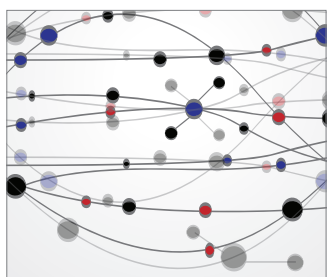

The Scientific World Journal
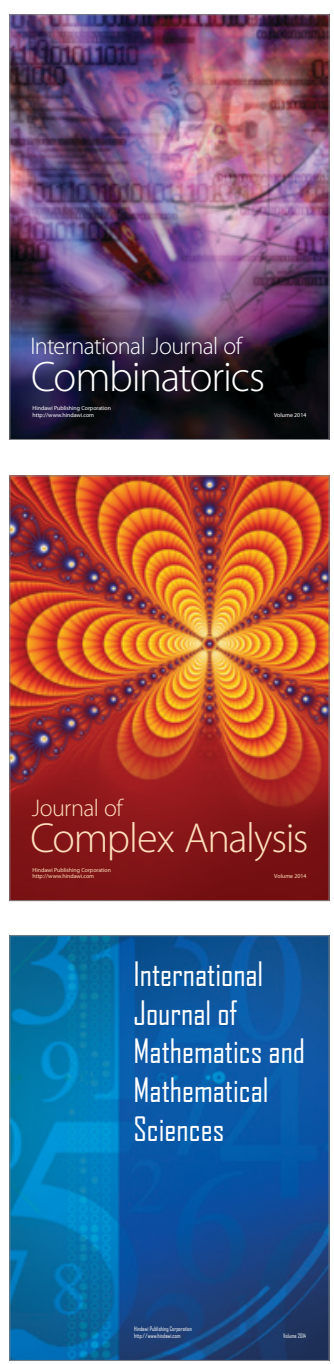
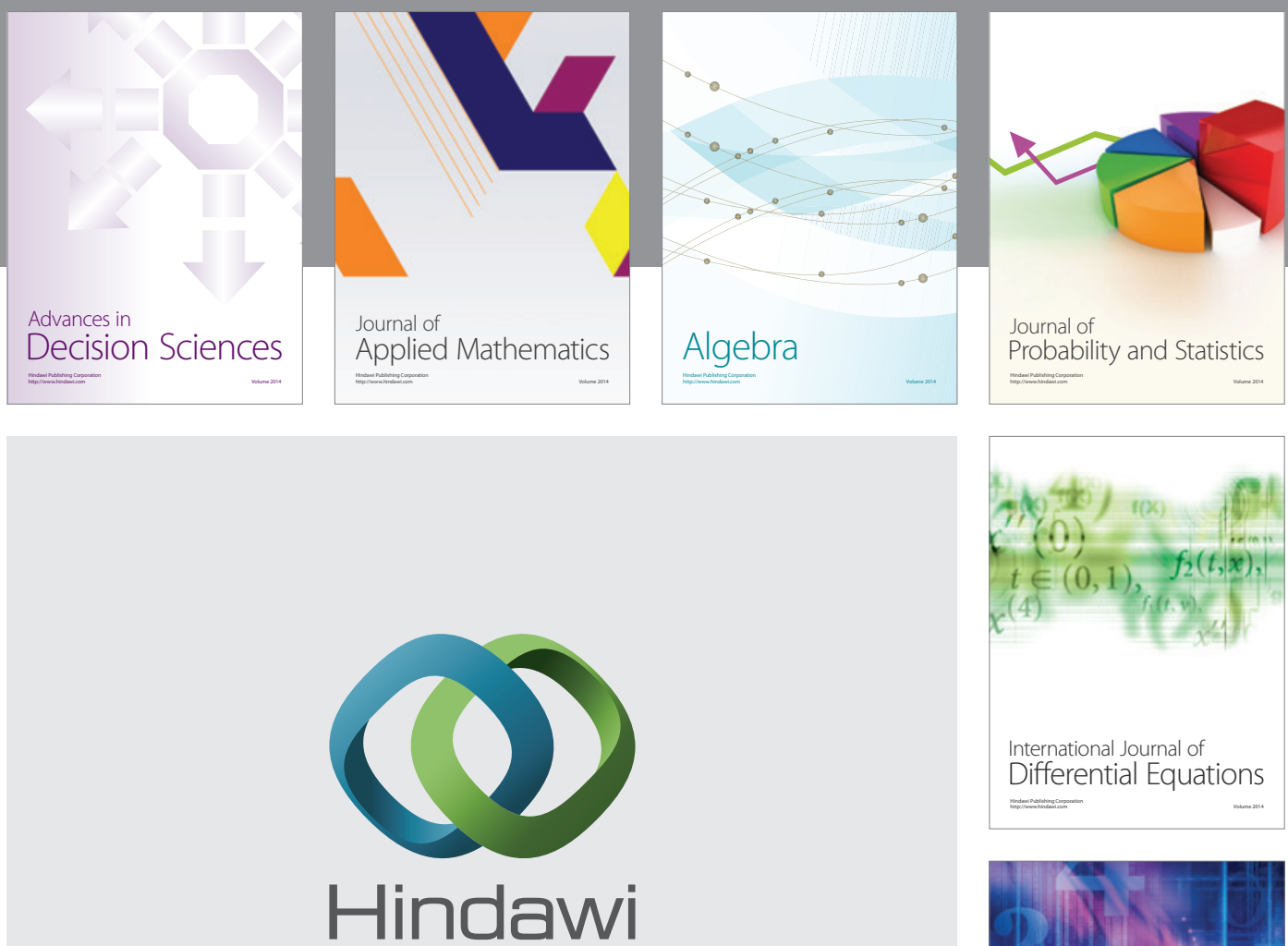

Submit your manuscripts at http://www.hindawi.com
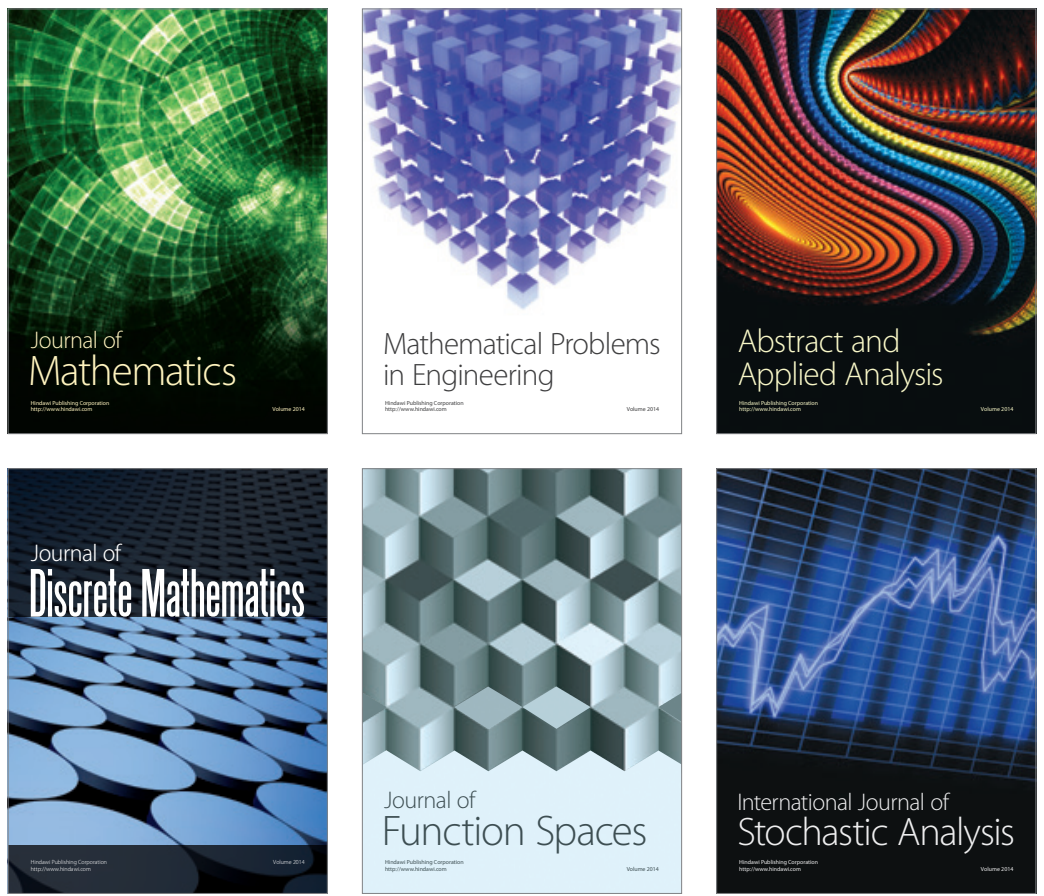

Journal of

Function Spaces

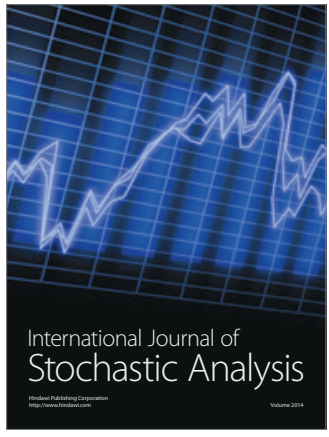

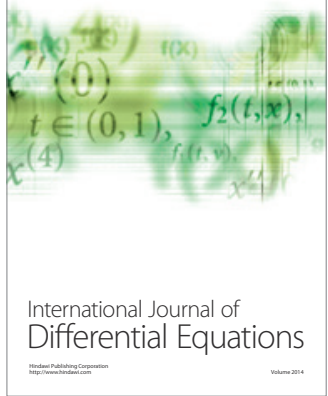
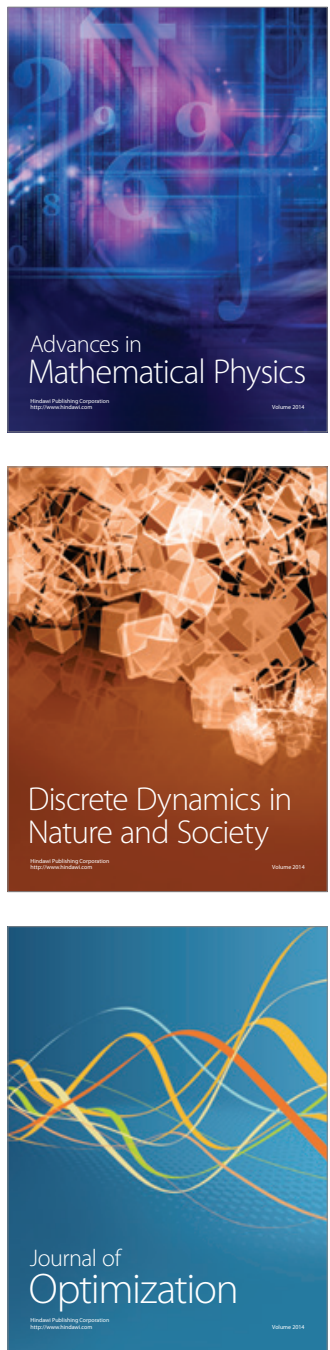